\title{
Assessment of diagnostic value of different methods (culture, PCR and biopsy) for the diagnosis of tuberculosis in patients with bronchial anthracosis
}

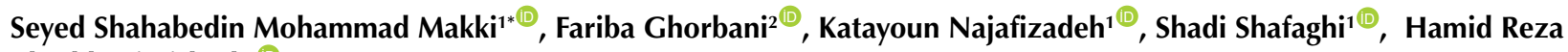 \\ Khoddami Vishteh ${ }^{1(\mathbb{D}}$
}

${ }^{1}$ Lung Transplant Research Center, Shahid Beheshti University of Medical Sciences, Tehran, Iran

${ }^{2}$ Tracheal Diseases Research Center, National Research Institute of Tuberculosis and Lung Diseases, Shahid Beheshti University of Medical Sciences; Tehran, Iran

\section{*Correspondence to}

Seyed Shahabedin Mohammad Makki,

Email: mohammad.makki@ sbmu.ac.ir, makkishahab@ yahoo.com

Received 3 Jan. 2020 Accepted 15 Mar. 2020 Published online 6 May 2020

Keywords: Tuberculosis, Anthracosis, Anthracofibrosis, bronchoalveolar lavage $(\mathrm{BAL})$ Polymerase chain reaction, Sensitivity, Specificity

\begin{abstract}
Introduction: Although several studies have shown the relationship between tuberculosis (TB) and anthracosis, few studies have investigated the diagnostic value of various methods of diagnosis of TB in these patients. Objectives: This study was conducted to investigate the diagnostic value of different methods including smear, culture and polymerase chain reaction (PCR) of bronchoalveolar lavage (BAL) sample and bronchial biopsy specimens in the diagnosis of TB in patients with bronchial anthracosis.

Patients and Methods: This descriptive cross-sectional study was performed on 67 patients referring for bronchoscopy, and bronchial anthracosis was an incidental finding. The BAL sample and patients' bronchial biopsies were examined for TB infection.

Results: The most affected areas by anthracosis were the right and the left upper lobes. The BAL smear and its culture were positive in $12(20 \%)$ and 10 patients (17\%), respectively. The PCR of BAL sample was positive in $37 \%$. Non-specific chronic inflammation followed by bronchial anthracosis was the most common pathologic findings. According to pathological examinations, nine patients (13\%) had TB. Sensitivity and specificity of the PCR versus culture were $90 \%$ and $70 \%$, respectively, with a diagnostic accuracy of $74 \%$. Sensitivity, specificity and diagnostic accuracy of the pathologic examination of biopsy specimen were $33 \%, 85 \%$ and $74 \%$, respectively.

Conclusion: Findings of this study showed that nearly one fifth of patients with bronchial anthracosis suffer from TB simultaneously. In these patients, PCR of the BAL and pathologic examination of the biopsy specimen had the same diagnostic accuracy, but the sensitivity of PCR was three times higher. However, application of PCR and pathologic examination does not seem to have more diagnostic advantages than BAL culture for the diagnosis of TB, although the results by PCR may help to diagnose the disease sooner.
\end{abstract}

\section{Introduction}

Anthracosis is a bronchoscopic finding which is characterized by formation of black pigments in the airways and mucous membranes of bronchi. Anthracosis can be the result of bronchial injury, metamorphosis, and obstruction. Pathologically, these lesions seem to be due to the deposition of silica, carbon and asbestos particles in the cytoplasm of mucosal and submucosal cells, as well as macrophages $(1,2)$.

Although initially it was thought that anthracosis had been a type of pneumoconiosis in coal workers (3), later this view was changed, and anthracosis or bronchial anthracofibrosis was considered as a different clinical syndrome (1), which could be due to the spread of urbanization and the presence of particles of carbon, asbestos and

\begin{abstract}
Key point
In patients with bronchial anthracosis who suffe from tuberculosis simultaneously, application of PCR and pathologic examination does not seem to have more diagnostic advantages than BAL culture for the diagnosis of TB, although the results by PCR may help to diagnose the disease sooner.
\end{abstract}

silica in the air. The prevalence of this disease is low in western countries, North America and Africa, with only a few reports (4-6), but more prevalent in Asian countries. Overall, the prevalence of simple anthracosis in patients who underwent bronchoscopy has been reported $3.4 \%-21 \%$ in various studies, while its cumulative prevalence is $5.7 \%$ (0.1$22.5 \%)(7,8)$. In a previous study from Iran, its prevalence is reported to be $1.1-31.89 \%$ (9).

\footnotetext{
Copyright (c) 2020 The Author(s); Published by Nickan Research Institute. This is an open-access article distributed under the terms of the Creative Commons Attribution License (http://creativecommons.org/licenses/by/4.0), which permits unrestricted use, distribution, and reproduction in any medium, provided the original work is properly cited.
} 
Pulmonary tuberculosis (TB) and AIDS are the most important public health problems in developing countries, because TB is a chronic disease and has its own treatment protocol, which imposes a high cost on the health care system. Several studies have reported the relationship between TB and anthracosis (9). In addition, the risk of $\mathrm{TB}$ and non-TB pneumonia is higher in patients with anthracosis (10). On the other hand, TB infection in these patients may have an atypical presentation, i.e., it does not have positive TB skin test (purified protein derivative; PPD) or does not form granuloma or may cause by certain subtypes of mycobacterium TB. Thus, the relationship between TB and anthracosis is complex, while there is a debate on their cause and effect relationship. Despite this complicated relationship, few studies have been carried out to assess the diagnostic value of different methods in the diagnosis of TB in patients with bronchial anthracosis $(11,12)$

\section{Objectives}

The aim of this study is to investigate the diagnostic value of different methods (including culture and polymerase chain reaction [PCR] of bronchoalveolar lavage [BAL] sample and pathologic examination of bronchial biopsy specimen) in the diagnosis of TB in these patients.

\section{Patients and Methods}

\section{Study design}

This descriptive cross-sectional study was performed on 67 patients who referred to the bronchoscopy department of Dr. Masih Daneshvari hospital in Tehran who had undergone bronchoscopy, and bronchial anthracosis (deposition of black pigments) or anthracotic bronchitis (deposition of black pigments with edema, deformity or stenosis of bronchi) were detected during the procedure. The study was explained to all patients before performing bronchoscopy. Patients' information including demographic data (age, gender, occupation and nationality), history of baking and its duration, fuel used for cooking and heating the house, smoking, symptoms and signs, history of TB and radiographic and CT scan findings were recorded. The location of the lesions in bronchoscopy was recorded. BAL samples were examined for smear, culture and PCR of TB and bronchial biopsy specimens were studied pathologically. However, due to some reasons such as bleeding etc., it was not possible to obtain BAL or tissue sample in all patients, additionally in some patients tissue sample was insufficient for evaluation.

\section{Ethical issues}

The study was approved by ethical committee of Shahid Beheshti University of Medical Sciences, since written consent was obtained from each patient before the study. In all stages of research, the tenets of the Declaration of Helsinki were observed. Additionally, This study was extracted from fellowship thesis of Seyed Shahabedin
Mohammad Makki at this university (Thesis\#141).

\section{Statistical analysis}

Data were analyzed by SPSS 13 software. Qualitative variables were described by frequency (percentage) and quantitative variables by using range, mean and standard deviation. In addition, the efficacy (sensitivity, specificity, positive predictive value, negative predictive value and diagnostic accuracy) of PCR of the BAL and the pathologic examination of the biopsy specimens versus the result of BAL culture for TB were calculated.

\section{Results}

Thirty-seven patients (55\%) were male and 30 (45\%) were female. The range and mean (SD) of their age were 41 87 years and $70 \pm 10$ years, respectively. Thirteen (19\%) were foreigners, while most of them (21 patients, 31\%) were farmers. Fourteen patients (21\%) were smokers, 10 (15\%) had a history of TB, seven of which were treated, and all patients had a negative sputum smear. Twenty-six patients (39\%) had a history of baking, and the range and mean (SD) of baking time were 3-58 years and $33 \pm 17$ years, respectively. Gas was the most commonly used fuel for both cooking and heating the house (73\% and 64\% respectively) (Table 1).

Cough was seen in 61 patients (91\%), sputum in 43 (64\%), dyspnea in 50 (75\%), fever in 25 (37\%), sweating in $34(51 \%)$, weight loss in 37 patients (55\%) and history of recurrent infection in four (6\%). On physical examination, 12 patients (18\%) had wheezing, eight (12\%) had rales, and $11(16 \%)$ patients had decreased breathing sounds.

The most common findings in chest X-ray (CXR) of patients were infiltration (in 22,33\%) and normal radiography was observed in $12(18 \%)$ patients. Similar to CXR, the most common finding in CT scan was infiltration (in 20,30\%), followed by collapse (in 14\%, 21\%). Only four (6\%) had normal CT scans (Table 2).

Table 3 shows the frequency of mucosal edema, anthracosis, and obstruction in different regions of the lower respiratory tract. The most common involved regions were the left upper lobe (LUL) and the right upper lobe (RUL), since each patient had average of 4.5 anthracotic regions in each lung (other than trachea and carina).

In 59 patients, the BAL sample was adequate for smear and culture. Twelve (20\%) were smear positive and 10 patients (17\%) had positive culture. Overall, in 10 patients (17\%) both smear and culture were positive. In addition,

Table 1. Type of fuel used for cooking and heating the house

\begin{tabular}{lcc}
\hline Type of fuel & Cooking & Heating the house \\
\hline Gas & $49(73 \%)$ & $43(64 \%)$ \\
Wood & $34(51 \%)$ & $30(45 \%)$ \\
Oil & $36(54 \%)$ & $42(63 \%)$ \\
Biomass & $26(39 \%)$ & $20(30 \%)$ \\
\hline
\end{tabular}


Table 2. Radiographic findings of patients' chest

\begin{tabular}{lcc}
\hline Findings & CXR & CT scan \\
\hline Collapse, infiltration and bronchiectasis & - & $2(3 \%)$ \\
Collapse and infiltration & $9(13 \%)$ & $13(19 \%)$ \\
Infiltration and bronchiectasis & - & $2(3 \%)$ \\
Collapse & $16(24 \%)$ & $14(21 \%)$ \\
Infiltration & $22(33 \%)$ & $20(30 \%)$ \\
Others & $8(12 \%)$ & $12(18 \%)$ \\
Normal & $12(18 \%)$ & $4(6 \%)$ \\
\hline
\end{tabular}

Table 3. Prevalence of involvement in different parts of the lower respiratory tract of patients

\begin{tabular}{lccc}
\hline Place & Mucosal edema & Anthracosis & Obstruction \\
\hline Trachea & $2(3 \%)$ & $2(3 \%)$ & $0(0 \%)$ \\
\hline Carina & $3(5 \%)$ & $6(9 \%)$ & $0(0 \%)$ \\
\hline RMB & $12(18 \%)$ & $26(39 \%)$ & $3(5 \%)$ \\
\hline RUL & $28(42 \%)$ & $47(70 \%)$ & $17(25 \%)$ \\
\hline RML & $21(31 \%)$ & $43(64 \%)$ & $15(22 \%)$ \\
\hline RLL & $18(27 \%)$ & $40(60 \%)$ & $8(12 \%)$ \\
LMB & $10(15 \%)$ & $23(34 \%)$ & $1(2 \%)$ \\
\hline LUL & $29(43 \%)$ & $56(84 \%)$ & $17(25 \%)$ \\
\hline Lingula & $22(33 \%)$ & $43(64 \%)$ & $15(22 \%)$ \\
\hline LLL & $14(21 \%)$ & $35(52 \%)$ & $8(12 \%)$ \\
\hline
\end{tabular}

RMB: Right main bronchus, RUL: Right upper lobe, RML: Right Middle lobe, RLL: Right lower lobe, LUL: Left upper lobe, LML: Left middle lobe, LLL: Left lower lobe, LMB: Left main bronchus.

only 25 patients had an adequate biopsy specimen for smear and culture since the latter was positive in only one case. In 60 patients, BAL sample was examined for PCR, which was positive in $22(37 \%)$ patients.

Overall, a total of 62 patients had various samples for pathologic examinations while nine of which (13\%) were inadequate. In 53 remaining patients, non-specific chronic inflammation followed by bronchial anthracosis were the most common pathologic diagnosis. In total, nine patients (13\%) had granuloma based on a pathological examination (Table 4).

Of 59 patients who had BAL culture, 53 patients had sufficient samples for PCR and 48 had sufficient samples for pathologic examination for TB. Compared to BAL culture, the sensitivity, specificity, and diagnostic accuracy of PCR was $90 \%, 70 \%$, and $74 \%$, respectively and pathologic examination was $33 \%, 85 \%$, and $74 \%$, respectively (Table 5).

\section{Discussion}

Findings of the study showed that, compared to BAL culture, the diagnostic accuracy of both PCR and pathologic examination for TB was similar, but the sensitivity of the PCR of the BAL sample was about three times more than that of BAL culture.

In most of the studies, association of anthracosis with TB is highlighted. The prevalence of TB in these patients

\begin{tabular}{lc} 
Table 4. Pathologic findings & No. (\%) \\
\hline Pathologic findings & $29(44)$ \\
\hline Chronic non-specific inflammation & $10(15)$ \\
\hline Bronchial anthracosis & $5(8)$ \\
\hline Granuloma & $2(3)$ \\
\hline Granuloma and bronchial anthracosis & $2(3)$ \\
\hline Small cell carcinoma & $1(2)$ \\
\hline Parenchymal anthracosis & $1(2)$ \\
\hline Fibrosis and parenchymal anthracosis & $1(2)$ \\
\hline Granuloma and parenchymal anthracosis & $1(2)$ \\
\hline Non-small cell carcinoma & $1(2)$ \\
\hline Granuloma and silicone nodule & $14(21)$ \\
\hline $\begin{array}{l}\text { Failure to sampling or insufficient sample for pathological } \\
\text { examination }\end{array}$ & \\
\hline
\end{tabular}

is reported as $31.88 \%$ by Mortazavi Moghaddam et al (9). Generally, the cumulative prevalence of TB in patients with anthracosis is reported $7.5 \%$ on average (7). However, whether anthracosis is the cause of $\mathrm{TB}$ or vice versa, it has not yet been addressed and long-term studies are necessary for this area.

In our study, $17 \%$ of patients were diagnosed with $\mathrm{TB}$ by BAL culture, while $37 \%$ of them were diagnosed with TB by PCR of BAL sample alone. Pathologic examinations revealed $13 \%$ of patients had $\mathrm{TB}$. Therefore, considering BAL culture as a gold standard for diagnosis of TB, PCR seems to have had more false positive results. In addition, sensitivity of PCR of the BAL sample in comparison with BAL culture was $90 \%$, its specificity was $70 \%$, and its diagnostic accuracy was $74 \%$. We also found that the sensitivity of tissue biopsy in diagnosis of TB was 33\% in comparison with the BAL culture, while its specificity was $85 \%$ and its diagnostic accuracy was $74 \%$. In other words, both PCR and pathologic examinations had the same diagnostic accuracy, but sensitivity of PCR was about three times higher. However, both methods had a low positive predictive value (about $40 \%$ or less), which means the positive test of each of these two methods indicates a $40 \%$ probability of TB in these patients.

In general, sensitivity of culture in diagnosis of $\mathrm{TB}$ was reported $80-85 \%$, and its specificity was $95 \%$. Although various studies have examined the prevalence of TB in anthracosis, only a few have assessed sensitivity and specificity of diagnostic methods of TB in these patients. Mirsadraee et al found acid fast bacilli in anthracosis and anthracofibrosis in $17.5 \%$ and $25 \%$ of patients respectively, and caseous granuloma in $20 \%$ and $17.5 \%$ of cases, respectively (13). Na et al reported sensitivity and specificity of PCR of bronchial washing specimen in the diagnosis of TB in 30 patients to be $89 \%$ and $86 \%$, respectively (12) which is higher than the results of our study. Different PCR methods in these two studies could explain this difference. Mirsadraee et al also examined different methods in diagnosis of $\mathrm{TB}$ in patients with 
Table 5. Clinical efficacy of PCR of BAL sample and pathological examination of biopsy specimen versus BAL culture for diagnosis of TB in patients with anthracosis*

\begin{tabular}{|c|c|c|c|c|}
\hline & \multicolumn{2}{|c|}{ PCR of BAL sample $(n=53)$} & \multicolumn{2}{|c|}{ Pathological examination of biopsy specimen $(n=48)$} \\
\hline & Positive & Negative & TB (granuloma) & Others \\
\hline Having TB (positive BAL culture) & 9 & 1 & 3 & 6 \\
\hline Not having TB (negative BAL culture) & 13 & 30 & 6 & 33 \\
\hline True positive & \multicolumn{2}{|c|}{$17 \%$} & \multicolumn{2}{|c|}{$5 \%$} \\
\hline False positives & \multicolumn{2}{|c|}{$25 \%$} & \multicolumn{2}{|c|}{$13 \%$} \\
\hline True negative & \multicolumn{2}{|c|}{$56 \%$} & \multicolumn{2}{|c|}{$69 \%$} \\
\hline False negative & \multicolumn{2}{|c|}{$2 \%$} & \multicolumn{2}{|c|}{$13 \%$} \\
\hline Sensitivity & \multicolumn{2}{|c|}{$90 \%$} & \multicolumn{2}{|c|}{$33 \%$} \\
\hline Specificity & \multicolumn{2}{|c|}{$70 \%$} & \multicolumn{2}{|c|}{$85 \%$} \\
\hline Positive predictive value & \multicolumn{2}{|c|}{$41 \%$} & \multicolumn{2}{|c|}{$33 \%$} \\
\hline Negative predictive value & \multicolumn{2}{|c|}{$97 \%$} & \multicolumn{2}{|c|}{$85 \%$} \\
\hline Diagnostic accuracy & \multicolumn{2}{|c|}{$74 \%$} & \multicolumn{2}{|c|}{$74 \%$} \\
\hline
\end{tabular}

* Of 67 patients, 59 patients had BAL culture, of which 53 were BAL PCR and 48 had a pathologic biopsy specimen for diagnosis of TB.

bronchial anthracosis, and reported the sensitivity and specificity of PCR compared to control group to be $66 \%$ and $100 \%$, respectively. However, they detected no significant difference between conventional methods and PCR in the diagnosis of TB in anthracosis patients. Finally, they suggested that PCR should only be performed if there is insufficient expertise to perform traditional techniques or if histopathological examination of the tissue is not possible due to bleeding (11).

Old age and female gender are reported as risk factors for anthracosis (4). Our study showed that the average age of patients was 70 and about half of them were women. Other studies have also suggested that anthracosis is the most common cause of chronic obstructive pulmonary disease in non-smoker females in developing countries (13-15). Like in other studies, only one-fifth of our patients were smokers. Old age of patients may be due to the long-term exposure required for deposition of specific environmental factors in airways. Additionally, physiologic changes in the airways of old people may be a predisposing factor for deposition of particles of biomass fuels. As many patients are non-smoker housewives in rural areas with a history of baking, the long-term history of baking is one of the risk factors for anthracosis and anthracofibrosis. In our study, $39 \%$ of patients had a history of baking. Hence, old age and history of baking or prolonged exposure to smoke or biomass fuels, particles of which mainly contain carbon, can help to recognize the pathophysiology of this disease to some extent. However, anthracosis may be an occupational disease, which is mentioned in some studies too (15-17).

\section{Conclusion}

Findings of this study revealed that nearly one-fifth of anthracotic patients suffered from TB simultaneously. In these patients, PCR of BAL sample and the pathologic examination of the biopsy sample have similar diagnostic accuracy (both 74\%), but sensitivity of PCR is three times higher (90\% versus 33\%). However, the predictive value of both methods is around $40 \%$, which means the positive result of these two methods indicates a $40 \%$ probability of TB in these patients. However, application of PCR and pathologic examination does not seem to have more diagnostic advantages than BAL culture for the diagnosis of TB, although the results by PCR may help to diagnose the disease sooner.

\section{Limitations of the study}

Some of our study limitations were small sample size, single center study, and not enough sample for pathologic examination in some patients.

Authors' contribution

All authors passed four criteria for authorship contribution based on recommendations of the International Committee of Medical Journal Editors. SSMM, KN, and FG designed the protocol of study. SSMM and FG developed the protocol and performed it. All authors performed critical revision of the manuscript for important intellectual content. Analysis of data was performed by HRKV. All authors read and approved the final paper.

Conflicts of interest

The authors declare that they have no conflict of interest.

Ethical considerations

Ethical issues (including plagiarism, data fabrication, double publication) have been completely observed by the authors.

Funding/Support

This study was supported by Shahid Beheshti University of Medical Sciences (Thesis\#141).

References

1. Chung MP, Lee KS, Han J, Kim H, Rhee $\mathrm{CH}$, Han $\mathrm{YC}$, et al. Bronchial stenosis due to anthracofibrosis. Chest 1998;113(2):344-50. doi: 10.1378/chest.113.2.344.

2. Ohshima S. Studies on pulmonary anthracosis. With special reference to the mineral constitution of intrapulmonary particulate pollutants in the human lung. Acta Pathol Jpn 1990;40(1):41-9. doi: 10.1111/j.1440-1827.1990.tb01527.x.

3. Fisher ER, Watkins G, Lam NV, Tsuda H, Hermann C, Johal $\mathrm{J}$, et al. Objective pathological diagnosis of coal workers' pneumoconiosis. JAMA 1981;245(18):1829-34. 
4. Hwang J, Puttagunta L, Green F, Shimanovsky A, Barrie J, Long R. Bronchial anthracofibrosis and tuberculosis in immigrants to Canada from the Indian subcontinent. Int J Tuberc Lung Dis 2010;14(2):231-7.

5. Ribeiro C, Guimarães $M$, Antunes A, Oliveira A, Neves $\mathrm{S}$, Almeida J, et al. "The black bronchoscopy": a case of airway soot deposition. J Bronchology Interv Pulmonol 2013;20(3):271-73. doi: 10.1097/LBR.0b013e31829add96.

6. Gómez-Seco J, Pérez-Boal I, Guerrero-González J, SáezNoguero F, Fernández-Navamuel I, Rodríguez-Nieto MJ. Antracofibrosis o antracoestenosis. Arch Bronconeumol 2012;48(4):133-36. doi: 10.1016/j.arbres.2011.07.006.

7. Mirsadraee M. Anthracosis of the lungs: etiology, clinical manifestations and diagnosis: a review. Tanaffos 2014;13(4):1.

8. Sigari N, Mohammadi S. Anthracosis and anthracofibrosis. Saudi Med J 2009;30(8):1063-6.

9. Mortazavi-Moghaddam SG, Saadatjoo SA. Bronchial anthracotic change in South Khorasan Province (Iran), emphasizing its association with tuberculosis. Iran J Med Sci 2014;39(5):418.

10. Wu HP, Pan YH, Hua CC, Shieh WB, Jiang BY, Yu TJ. Pneumoconiosis and liver cirrhosis are not risk factors for tuberculosis in patients with pulmonary infection. Respirology 2007;12(3):416-9. doi: 10.1111/j.1440-1843.2007.01033.x.

11. Mirsadraee M, Shafahie A, Khakzad MR, Sankian M. Value of the polymerase chain reaction method for detecting tuberculosis in the bronchial tissue involved by anthracosis.
J Bronchology Interv Pulmonol 2014;21(2):131-34. doi: 10.1097/LBR.0000000000000063.

12. Na J-O, Lim C-M, Lee S-D, Koh Y-S, Kim W-S, Kim D-S, et al. Detection of Mycobacterium tuberculosis in bronchial specimens using a polymerase chain reaction in patients with bronchial anthracofibrosis. Tuberc Respir Dis (Seoul) 2002;53(2):161-72.

13. Mirsadraee MH, Asnashari AK, Attaran DM. Tuberculosis in patients with anthracosis of lung underlying mechanism or superimposed disease. Iran Red Crescent Med J 2011;13(9):670-3. doi: 10.5812/kowsar.20741804.2247.

14. Kim J, Park J, Kang M, Yu C, Kim Y, Han S, et al. Endobronchial anthracofibrosis is causally associated with tuberculosis. Korean J Intern Med 1996;51:351-57.

15. Gupta A, Shah A. Bronchial anthracofibrosis: an emerging pulmonary disease due to biomass fuel exposure. Int J Tuberc Lung Dis 2011;15(5):602-12. doi: 10.5588/ijtld.10.0308

16. Ghanei M, Aslani J, Peyman M, Asl MA, Pirnazar O. Bronchial anthracosis: a potent clue for diagnosis of pulmonary tuberculosis. Oman Med J 2011;26(1):19-22. doi: 10.5001/ omj.2011.05.

17. Wynn GJ, Turkington PM, O'Driscoll BR. Anthracofibrosis, bronchial stenosis with overlying anthracotic mucosa: possibly a new occupational lung disorder: a series of seven cases From one UK hospital. Chest 2008;134(5):1069-73. doi: 10.1378/ chest.08-0814 